\title{
Index to Volume 121
}

\section{Compiled by Leslie Cody}

Abies balsamea, 327,329,362

$$
\text { lasiocarpa, } 156
$$

Absinth, 184

Acalypha virginica, 184

Accipiter cooperii, 216 gentilis, 329,413

Accipiter gentilis, Exploits a Beagle Hound, Canis familiaris, as a "Beater" to Catch a Snowshoe Hare, Lepus americanus, Northern Goshawk, 329

Acer rubrum, 385

saccharinum, 385

saccharum, 256,304,420

spicatum, 359

Achillea millefolium, 77 millefolium ssp. millefolium, 184

Acipenser brevirostrum, 176

Actitis hypoleucos, 413

Advice to Contributors, 478

Aechmophorus clarkii, 50 occidentalis, 50

Agelaius phoeniceus, 245

Agrimonia gryposepala, 184

Agrimony, Hooked, 184

Agrostis gigantea, 184

Albert, A.J., 92

Alberta, The Influence of Air Pollution on Corticolous Lichens near the Strathcona Industrial Area, 17

Alberta, Twenty-Four-Hour Activity Budgets of Mule Deer, Odocoileus hemionus, in the Aspen Parkland of Eastcentral, 299

Alca torda, 275,289

Alces alces, 156,359,391,416 alces americana, 265 americanus, 121

Alces alces, Winter Browse Use in Central Labrador, Moose, 359

Alder, 82,329 Mountain, 81

Alectoria spp., 380

Alfalfa, 184 Alisma gramineum, 165 plantago-aquatica, 165

Allan, M., 212

Alliance of Natural History Museums of Canada Annual Meeting and Awards, 349

Alnus sp., 82,329 crispa, $81,297,362$

Alopex lagopus, 73,308,322,330

Ambrosia artemisiifolia, 184

Amelanchier sp., 86 arborea, 89 arborea var. arborea, 203

canadensis, 89

interior, 90

intermedia 89 laevis, 89,203

spicata, 90

stolonifera, 203

x grandiflora, 89

Amelanchier intermedia, Escaped from Cultivation in Eastern Ontario, Serviceberry, 89

American-aster, White Heath, 204

Ammodramus bairdii, 244

henslowii, 244

leconteii, 244

savannarum, 244

Ammodytes spp., 285

hexapterus, 325

Amnicola limosa, 92

Anaphalis margaritacea, 184

Anas acuta, 245

americana, 245,325,376

clypeata, 245

crecca, 326

discors, 245

platyrhynchos, 130,245

strepera, 245

Anastrophyllum assimile, 26

Anderson, R.C., R. Shimek, J.A. Cosgrove, and S. Berthinier. Giant Pacific Octopus, Enteroctopus dofleini, Attacks on Divers, 423

Andropogon gerardii, 203

Anemone canadensis, 184 cylindrica, 203

Anemone, Canada, 184

Angelica genuflexa, 421

Anodonta implicate, 93

Antennaria howellii ssp. petaloidea, 203 neglecta, 184 parlinii, 184 parlinii ssp. fallax, 203

Anthelia julacea, 26 juratzkana, 26

Anthus spragueii, 244

Apalone spinifera, 178

Apocynum androsaemifolium, 203

Aquila chryseatos, 156

Aquilegia canadensis, 203

Arabis divaricarpa, 203 hirsuta var. pycnocarpa, 203

holboellii var. retrofracta, 203

Arctium minus ssp. minus, 184

Arctostaphylos uva-ursi, 203

Ardea herodias, 426

Aries, C.E., 325

Artemia, 130

Artemisia spp., 134 absinthium, 184 campestris $\mathrm{ssp}$. borealis var. scouleriana, 203

Arthonia patellulata, 18 
Asclepias exaltata, 203

syriaca, 184,203

tuberosa ssp. tuberosa, 203

Ash, Black, 385

Mountain, 359

White, 304

Asio flammeus, 245

Aspen, 256,297,304

Quaking, 150

Trembling, 18,191,261,327

Aster ssp., 182

cordifolius, 184

curtus, 40

ericoides, 184

lanceolatus, 184

lateriflorus, 184

novae-angliae, 185

ontarionis, 186

simplex, 184

umbellatus, 184

urophyllus, 184

Aster, Arrow-leaved, 184

Calico, 184

Flat-topped White, 184

Heart-leaved, 184

Heath, 184

New England, 185,204

Panicled, 184

Sky-blue, 204

Western Willow, 166

White-top, 40

Aster, Sericocarpus rigidus, a Threatened Species from the Garry Oak Ecosystem in British Columbia, MicroPropagation of White-top, 40

Atlas of amphibians and reptiles of Quebec, 2007 inventory, 234

Avens, White, 184

Yellow, 184

Avocet, American, 245

Aythya affinis, 245

americana, 245

collaris, 245

valisineria, 245

Badger, American, 150

European, 265

Ballard, W.B., 71

Banville, D., 402

Barbarea vulgaris, 184

Barbilophozia floerkei, 26

hatcheri, 26

kunzeana, 26

lycopodioides, 26

voerkei, 27

Barley, Wild, 166

Bartramia longicauda, 245

Basil, Wild, 184

Bat, 385

Big Brown, 120

Eastern Red, 124,386

Hoary, 124,208,386

Little Brown, 57,208,386,420

Long-legged, 124

Northern Long-eared, 208,386

Red, 208
Silver-haired, 121

Western Long-eared, 124

Bat, Myotis lucifugus, in Manitoba and Northwestern Ontario, Recent Distribution Records of the Little Brown, 57

Bat, Myotis lucifugus, Observation of Foliage-roosting in the Little Brown, 420

Bat, Myotis septentrionalis (Chiroptera: Vespertilionidae), on Prince Edward Island: First Records of Occurrence and Over-Wintering, Northern Long-eared, 208

Bay, California, 305

Bazzania pearsonii, 26

Bear, 385 tricrenata, 26

American Black, 120,387

Black, 156,266,330,362,430

Grizzly, 120,156

Polar, 120

Bearberry, Red, 203

Beardtongue, Hairy, 203

Beaver, 271,330,385,438

American, 120,391

Giant, 330

Beaver, Castoroides ohioensis, Remains in Canada and an Overlooked Report from Ontario, Giant, 330

Bedstraw, Licorice, 203 Northern, 203

Small, 184

Beech, American, 304,385

Beetle, 182

Bennett, B.A., 295

Berthinier, S., 423

Betula alleghaniensis, 385 glandulosa, 81,297 papyrifera, 81,304,327,359

Bindweed, Black, 184 Field, 184 Low False, 203

Birch, Dwarf, 81 White, 81,304,327,359 Yellow, 385

Bison bison, 121,130,192 bison athabascae, 124

Bison, 192

American, 121,130

Wood, 124

Bittern, American, 245

Blackbird, Brewer's, 243

Red-winged, 242

Yellow-headed, 245

Blarina brevicauda, 206,386

Blatt, S.E., 182

Blepharostoma trichophyllum, 27 trichophyllum ssp. trichophyllum , 26

Blomme, C.G., 436

Bluegrass, Alpine, 134

Bluejoint, Canada, 184,433

Bluestem, Big, 203 Little False, 204

Blueweed, 184

Bobcat, 437

Bobolink, 243

Bombycilla cedrorum, 244

Bonasa umbellus, 329

Bond, A.L. and A.W. Diamond. Abandoned Seabird Eggs as a Calcium Source for Terrestrial Gastropods, 433 
Boneset, 184

Boreal Dip Net/L'epuosette boreal, The, 234,349

Bos taurus, 72

Botaurus lentiginosus, 245

Bouteloua curtipendula, 71 gracilis, 71

Branta canadensis, 245,313 canadensis maxima, 316 canadensis moffitti, 316

Branta canadensis, Management Implications of Molt Migration by the Atlantic Flyway Resident Population of Canada Geese, 313

Brazil, J., 81

Brice, J.S., 46

British Columbia, Cowbane, Oxypolis occidentalis, A New Native Vascular Plant Species for the Queen Charlotte Islands, 421

British Columbia, Micro-Propagation of White-top Aster, Sericocarpus rigidus, a Threatened Species from the Garry Oak Ecosystem in, 40

British Columbia, 1997 2005, Translocation and Recovery Efforts for the Telkwa Caribou, Rangifer tarandus caribou, Herd in Westcentral, 155

British Columbia's Gulf Island Waters, A River Otter's, Lontra canadensis, Capture of a Double-crested Cormorant, Phalacrocorax auritus, in, 325

British Columbia, The Hepatic Flora and Floristic Affinity of Hepatics Around Takakia Lake, Queen Charlotte Islands, 24

British Columbia, The Rumsfeld Paradigm: Knowns and Unknowns in Characterizing Habitats Used by the Endangered Sharp-tailed Snake, Contia tenuis, in Southwestern, 142

Brome, Smooth, 184

Bromus inermis ssp. pumpellianus, 183

Brown, J.A., D.F. McAlpine, and R. Curley. Northern Long-eared Bat, Myotis septentrionalis (Chiroptera: Vespertilionidae), on Prince Edward Island: First Records of Occurrence and Over-Wintering, 208

Bruce, L., 212

Bryoria spp., 18,303,380

Bubo virginianus, 437

Bubulcus ibis, 50,330

Bucephala albeola, 370,375

Bucephala albeola) After Twilight in Winter: An Anti-Predation Tactic?, Offshore Flight of Buffleheads, 375

Bucephala albeola: Autumn Arrivals in Shoal Harbour Sanctuary, Vancouver Island, in Relation to Freeze-up, The Punctual Bufflehead, 370

Buchlöe dactyoides, 72

Buffalo-berry, Russet, 204

Buffalograss, 72

Bufflehead, 370,375

Bufflehead, Bucephala albeola: Autumn Arrivals in Shoal Harbour Sanctuary, Vancouver Island, in Relation to Freeze-up, The Punctual, 370

Buffleheads (Bucephala albeola) After Twilight in Winter: An Anti-Predation Tactic?, Offshore Flight of, 375

Buller, S.A., 430

Bulrush, Great, 166

Hardstem, 54

Small-fruited, 166

Bunchgrass, 134

Bunting, Lark, 242

Snow, 310
Bur-Reed, 166

Burdock, Common, 184

Burrograss, 71

Buteo lagopus, 330

lineatus, 216

platypterus, 216

regalis, 245

swainsoni, 245

Buttercup, Field, 184

Kidney-leaf, 184

Labrador, 204

Thread-leaved, 166

Caenestheriella, 129

belfragei, 131

gynecia, 128

setosa, 130

Caenestheriella gynecia (Crustacea: Conchostraca), in New York and New Jersey, State Records and Habitat of Clam Shrimp, 128

Calamagrostis canadensis, 183,433

Calamospiza melanocorys, 244

Calcarius ornatus, 244

Calidris alpina, 376

Calypogeia azurea, 26 integristipula, 26 neesiana, 26

Calystegia spithamaea ssp. spithamaea, 203

Campion, Bladder, 184

Canada and an Overlooked Report from Ontario, Giant Beaver, Castoroides ohioensis, Remains in, 330

Canada, Arboreal Late Summer Courtship Behaviour of Maritime Garter Snake, Thamnophis sirtalis pallidulus, in Dartmouth, Nova Scotia, 210

Canada, Before and After the Application of the Herbicide Magnacide, An Inventory of the Aquatic and Subaquatic Plants in SASKWater Canals in Central Saskatchewan, 164

Canada Geese, Branta canadensis, Management Implications of Molt Migration by the Atlantic Flyway Resident Population of, 313

Canada, Human-assisted Movements of Raccoons, Procyon lotor, and Opossums, Didelphis virginiana, between the United States and, 212

Canadian Association of Herpetologists/Association Canadienne des Herpetologists Bulletin 14(2) Spring 2007, 234

Canadian Field-Naturalist, Advice for Contributors to The, $118,238,358$

Canis familiaris, 268,329

latrans, $71,120,150,156,268,328,364,392,397,426$, 430,437

lupus, 120,156,245,256,364,386,397,430,437

lupus baileyi, 430

rufus, 430

Canis familiaris, as a "Beater" to Catch a Snowshoe Hare, Lepus americanus, Northern Goshawk, Accipiter gentilis, Exploits a Beagle Hound, 329

Canis latrans, in Urbanized Eastern Massachusetts, Movements of Transient, 364

Canis latrans, Pack, Social and Play Behavior in a Wild Eastern Coyote, 397

Canis latrans, predation on Great Black-backed Gull, Larus marinus, eggs on Boot Island National Wildlife Area, Nova Scotia, Details of Eastern Coyote, 426 
Canis lupus, Behavior in Areas of Frequent Human Activity, Wolf, 256

Canis lupus, Pups into Another Wolf Pack, Attempt to CrossFoster Gray Wolf, 430

Canvasback, 245

Capelin, 285

Capsella bursa-pastoris, 78,184

Cardamine pensylvanica, 78 umbellata, 24

Carduelis tristis, 245

Carex spp., 134,187,359

aurea, 184

backii, 203

communis, 184

cusickii, 421

debilis, 184

foenea, 184

gracillima, 184,203

granularis, 184

laxiflora, 184

molesta, 203

muehlenbergii var. muehlenbergii, 203

normalis, 184

pensylvanica, 203

richardsonii, 203

rostrata, 166

scoparia, 184

siccata, 203

stylosa, 421

tonsa var. rugosperma, 203

vulpinoidea, 184

Caribou, 82,121,327,379,385

Telkwa, 155

Woodland, 96,155,360,389

Caribou, Rangifer tarandus caribou, Herd in Westcentral British Columbia, 1997-2005, Translocation and Recovery Efforts for the Telkwa, 155

Caribou, Rangifer tarandus, Track Decadal Changes in Arctic Tundra Vegetation, Diets of Overwintering, 379

Carrot, Wild, 184

Carthamus tinctorius, 44

Cartier, I., 402

Castor canadensis, 120,271,330,391,438

Castoroides, 330 ohioensis, 330

Castoroides ohioensis, Remains in Canada and an Overlooked Report from Ontario, Giant Beaver, 330

Cat, Domestic, 268

House, 208

Wild, 385

Catbird, Gray, 244

Catling, P.M. and B.A. Bennett. Discovery of a Possibly Relict Outbreeding Morphotype of Sparrow's-egg Lady's-slipper Orchid, Cypripedium passerinum, in Southwestern Yukon, 295

Catling, P.M. and B. King. Natural Recolonization of Cultivated Land by Native Prairie Plants and its Enhancement by Removal of Scots Pine, Pinus sylvestris, 201

Catling, P.M. and G. Mitrow. Serviceberry, Amelanchier intermedia, Escaped from Cultivation in Eastern Ontario, 89

Cattail, 53

Common, 166

Narrowleaf, 53

Ceanothus americanus, 203
Cedar, Eastern White, 304,385

White, 431

Celery, Wild, 164

Cepaea hortensis, 434

Cephalozia ambigua, 26

bicuspidata, 26

leucantha, 26

lunulifolia, 26

macounii, 26

Cepphus grylle, 275,289

Cerastium fontanum ssp. triviale, 184 vulgatum, 78

Ceratophyllum demersum, 93,165

Cervus canadensis, 192 elaphus, 62,121,156,214,334,416

elaphus nelsoni, 133,416

Cervus elaphus nelsoni, Populations, Colonization of NonTraditional Range in Dispersing Elk, 133

Cervus Elaphus, Using Tooth Wear/Eruption Patterns and Counts of Annuli in Tooth Cementum, Correlation Between Age Estimates for Elk, 214

Cervus elaphus, with Telezol and Xylazine and Reversal with Tolazine or Yohimbine, Immobilization of Elk, 62

Cetraria spp., 380

Chabot, D. and R. Miller. A Rare Case of Completely Ambicoloured Atlantic Halibut, Hippoglossus hippoglossus, from the Lower St. Lawrence Estuary, Quebec, 35

Chara, 166

Charadrius vociferus, 130

Chelydra serpentina, 130,178

Chelydra serpentina, Hatchlings in New York and New Hampshire, Post-Emergence Movements and Overwintering of Snapping Turtle, 178

Cheney, M. and K.L. Marr. Cowbane, Oxypolis occidentalis, A New Native Vascular Plant Species for the Queen Charlotte Islands, British Columbia, 421

Chenopodium simplex, 184

Cherry, Black, 203

Choke, 203

Clammy Ground, 184,203

Pin, 359

Susquehanna Sand, 203

Chhin, S. and G.G. Wang. Growth of White Spruce, Picea glauca, Seedlings in Relation to Microenvironmental Conditions in a Forest-Prairie Ecotone of Southwestern Manitoba, 191

Chickadee, Black-capped, 428 Chestnut-backed, 428

Chickweed, 185 Common Mouse-ear, 184

Chicory, 184

Chipmunk, 385

Eastern, 271,389

Least, 120

Chisholm, B., 214

Chlidonias niger, 50

Chondestes grammacus, 244

Chordeiles minor, 245

Chrysanthemum leucanthemum, 184

Chrysemys picta, 178

Chrysops sp., 257

Chubbs, T.E., 359

Chubbs, T.E. and F.R. Phillips. A Tribute to Neal Philip Perry Simon 1973-2006, 96 
Chubbs, T.E. and F.R. Phillips. Winter Occurrences of Ivory Gulls, Pagophila eburnea, in Inland Labrador, 327

Chubbs, T.E. and J. Brazil. The Occurrence of Muskoxen, Ovibos moschatus in Labrador, 81

Cichorium intybus, 184

Cinquefoil, Downy, 184

Erect, 184

Old Field, 184,203

Rough, 184

Silvery, 184

Tall, 203

Cionella lumbria, 434

Circus cyaneus, 245,330

Cirsium arvense, 184,302

Cistothorus palustris, 244 platensis, 244

Cladina spp., 380

Clark in 1804-1806, Were Native People Keystone Predators? A Continuous-Time Analysis of Wildlife Observations Made by Lewis and, 1

Clemmys guttata, 178

Clethrionomys gapperi, 271,390

Clinopodium vulgare, 184

Clover, Alsike, 185

Red, 185

Smaller Hop, 185

White Sweet, 166,184

Yellow, 185

Yellow Sweet, 184

Clupea harengus, 433 pallasii, 325

Clyde, K.J., 422

Coad, B.W., Review by, 102

Coccyzus erythropthalmus, 245

Cod, 285

Cody Receives Honorary Doctorate of Science from McMaster University, June 5, 2007, Bill, 233

Cole, M.J., 379

Colpitts, D.W., 426

Columbine, Red, 203

Comandra umbellata ssp. umbellata, 203

Comptonia peregrina, 203

Condylura cristata, 206

Condylura cristata) in Northeastern Vermont, A High Elevation Record of the Star-nosed Mole, 206

Contia tenuis, 124

Contia tenuis, in Southwestern British Columbia, The Rumsfeld Paradigm: Knowns and Unknowns in Characterizing Habitats Used by the Endangered Sharptailed Snake, 142

Convolvulus arvensis, 184

Conyza canadensis, 184

Cook, F.R., Reviews by, 100,338,339,342,343,347,439,443, 444,448

Cool, N., 214

Coot, American, 50,245

Copperleaf, 184

Cormorant, 274

Double-crested, 275,288,325,426

Great, 275,288

Pelagic, 325

Cormorant, Phalacrocorax auritus, in British Columbia's Gulf Island Waters, A River Otter's, Lontra canadensis, Capture of a Double-crested, 325
Cornus racemosa, 203

stolonifera, 359

Corvus brachyrhynchos, 150,427 corax, 150,427

Cosgrove, J.A., 423

Cota-Sånchez, J.H. and K. Remarchuk. An Inventory of the Aquatic and Subaquatic Plants in SASKWater Canals in Central Saskatchewan, Canada, Before and After the Application of the Herbicide Magnacide, 164

Cott, P.A. and N.J. Mochnacz. Bull Trout, Salvelinus confluentus, and North American Porcupine, Erethizon dorsatum, Interaction in the Mackenzie Mountains, Northwest Territories, 437

Cotter, R., 287

Cotter, R. and J.-F. Rail. Third Census of Seabird Populations of the Gaspé Peninsula, Québec, 2002, 274

Cottongrass, 134

Couchgrass, 184

Cougar, 120,437

Cow, 72

Cowbane, 421

Cowbane, Oxypolis occidentalis, A New Native Vascular Plant Species for the Queen Charlotte Islands, British Columbia, 421

Cowbird, Brown-headed, 239

Cowbird, Molothrus ater, Parasitism and Abundance in the Northern Great Plains, Brown-headed, 239

Coyote, 71,120,150,156,268,328,364,392,397,430,437 Eastern, 426

Coyote, Canis latrans, Pack, Social and Play Behavior in a Wild Eastern, 397

Coyote, Canis latrans, predation on Great Black-backed Gull, Larus marinus, eggs on Boot Island National Wildlife Area, Nova Scotia, Details of Eastern, 426

Coyotes, Canis latrans, in Urbanized Eastern Massachusetts, Movements of Transient, 364

Cranberry, 257

Crane, Whooping, 257

Crane's Bill, Bicknell's, 184

Crataegus, 86

Crins, W.J., Reviews by, 336,344

Crossman, L.M., 92

Crow, American, 150,427

Crowberry, Black, 81

Crowder, A., R. Harmsen, and S.E. Blatt. Notes on Succession in Old Fields in Southeastern Ontario: the Herbs, 182

Crowell, M., 265

Cuckoo, Black-billed, 245

Curley, R., 208

Cyathura carinata, 168 polita, 168

Cyathura polita in the Saint John River Estuary, New Brunswick: a Species at the Northern Extent of its Range, Life History of the Marine Isopod, 168

Cyperus houghtonii, 203 lupulinus ssp. lupulinus, 203

Cypripedium passerinum, 295

Cypripedium passerinum, in Southwestern Yukon, Discovery of a Possibly Relict Outbreeding Morphotype of Sparrow's-egg Lady's-slipper Orchid, 295

Cystophora cristata, 392

Cyzicus, 129

Dactylis glomerata, 184 
Dadswell, M.J., 168

Daisy, Ox-eye, 184

Dalén, L., A. Götherström, T. Meijer, and B. Shapiro. Recovery of DNA from Footprints in the Snow, 321

Dandelion, 182

Danthonia spicata, 184,203

Daucus carota, 184

Davies, C., 212

Davy, C.M. and E.E. Fraser. Observation of Foliage-roosting in the Little Brown Bat, Myotis lucifugus, 420

Deer, 151,385

Mule, 121,156,271,299

Red, 416

White-tailed, 121,139,257,265,302,333,391,412,426, 430

Deer, Odocoileus hemionus, in the Aspen Parkland of Eastcentral Alberta, Twenty-Four-Hour Activity Budgets of Mule, 299

Deer, Odocoileus virginianus, Fawn in Southcentral Wisconsin, A Late Born White-tailed, 333

Deer, Odocoileus virginianus, Seasonal Variation in Sex Ratios Provides Developmental Advantages in White-tailed, 412

Deermouse, North American, 120

Northwestern, 120

Dendragapus canadensis, 329

Dendroica petechia, 244

Deveau, A.E., 85

Devil, King, 184

Dezhi, L. and Q. Aili, Reviews by, 445

Dezhi, L., Q. Aili and Q. Hong, Reviews by, 108,111

Dezhi, L., Review by, 225

Diamond, A.W., 433

Dianthus armeria, 184

Dichanthelium columbianum, 203

depauperatum, 203

linearifolium, 203

perlongum, 203

sabulorum var. thinium, 203

Dickcissel, 245

Dicrostonyx groenlandicus, 120 nunatakensis, 120

Didelphis virginiana, 212,416

Didelphis virginiana, between the United States and Canada, Human-assisted Movements of Raccoons, Procyon lotor, and Opossums, 212

Diplophyllum albicans, 26 imbricatum, 26

plicatum, 26

taxifolium, 26

Dipoides, 331

Ditchkoff, S.S., 412

Dock, Curly, 184

Dodecatheon jeffreyi, 24

Dog, Domestic, 268,

Dogbane, Spreading, 203

Dogwood, Gray, 203

Red-osier, 359

Dolichonyx oryzivorus, 245

Donovan, D., 212

Dove, Mourning, 245,436

Dove, Zenaida macroura, Fish-line Entanglement of Nesting Mourning, 436

Draud, M., 178

Dropseed, 184
Dubois, J.E. and K.M. Monson. Recent Distribution Records of the Little Brown Bat, Myotis lucifugus, in Manitoba and Northwestern Ontario, 57

Duck, Harlequin, 82

Ring-necked, 245

Ruddy, 50

Duck Nests, Do Repugnant Scents Increase Survival of Ground Nests? A Test with Artificial and Natural, 150

Duckweed, 166

Dumetella carolinensis, 244

Dunlin, 376

Dunn, J., 313

Duskysnail, Squat, 92

Duskysnail, Lyogyrus granum (Mollusca), Widespread in the Hampton Marsh, New Brunswick, A Freshwater Hydrobiid, cf. the, 92

Eagle, Bald, 376

Golden, 156

Echinacea pallida, 44

Echinochloa crusgalli, 183

Echinochloa, 184

Echium vulgare, 184

Editor's Report for Volume 102 (2006), 235

Eel-grass, American, 164

Egret, Cattle, 50,330 Snowy, 50

Egretta caerulea, 54 thula, 50

Ehret, D.L., 40

Eider, Common, 275,288,308 Pacific Common, 308

Eider, Somateria mollissima, Nests on a Central Beaufort Sea Barrier Island: A Case Where No One Wins, Depredation of Common, 308

Elderberry, Red, 426

Eleocharis acicularis, 166 palustris, 166

Elk, 62,121,133,156,192,214,334,416

Elk, Cervus elaphus nelsoni, Populations, Colonization of Non-Traditional Range in Dispersing, 133

Elk, Cervus Elaphus, Using Tooth Wear/Eruption Patterns and Counts of Annuli in Tooth Cementum, Correlation Between Age Estimates for, 214

Elk, Cervus elaphus, with Telezol and Xylazine and Reversal with Tolazine or Yohimbine, Immobilization of, 62

Elliptio complanata, 93

Elm, American, 385

Elodea canadensis, 164

Elsinger, M., E. Burrell, N. deBruyn, K. Tanasichuk, and K. Timoney. The Influence of Air Pollution on Corticolous Lichens near the Strathcona Industrial Area, Alberta, 17

Elymus repens, 183 trachycaulon ssp. trachycaulon, 183 trachycaulus ssp. subsecundus, 203 trachycaulus ssp. trachycaulus, 184 trachycaulus ssp. unilaterale, 203

Empetrum nigrum, 81

Empidonax minimus, 244 traillii, 244

Emydoidea blandingii, 178

Engelstoft, C., 142

Enhydra lutra, 376 
Enteroctopus dofleini, 423

Enteroctopus dofleini, Attacks on Divers, Giant Pacific Octopus, 423

Epigaea repens, 79

Epilobium spp., 359

ciliatum, 166

delicatum, 24

hirsutum, 184

Eptesicus fuscus, 120

Equisetum sp., 359

arvense, 184

hyemale, 184

palustre, 166

Eremontus myriocarpus, 26

Eremophila alpestris, 244

Erethizon dorsatum, 120,266,391,437

Erethizon dorsatum, Interaction in the Mackenzie Mountains, Northwest Territories, Bull Trout, Salvelinus confluentus, and North American Porcupine, 437

Erigeron annuus, 184 peregrinus, 24 philadelphicus ssp. philadelphicus, 184 strigosus, 184

Eriophorum spp., 134 angustifolium, 24

Ermine, 120,385

Erysimum cheiranthoides, 78

Eumeces, 217 fasciatus, 216 okadae, 216

Eumeces fasciatus, Predator-Prey Interaction Between an American Robin, Turdus migratorius, and a Fivelined Skink, 216

Euonymus alata, 85

americana, 85

atropurpurea, 85

europaea, 85

fortunei, 85

nana, 85

obovata, 85

occidentalis, 85

Euonymus europaea L. (Celastraceae): A Newly Naturalized Shrub in Nova Scotia, The Spindle Tree, 85

Eupatorium maculatum, 184 perfoliatum, 184 rugosum, 184

Euphagus cyanocephalus, 245

Euphorbia helioscopa, 78

Euthamia graminifolia, 184

Everlasting, Pearly, 184

Evernia mesomorpha, 18

Fagus grandifolia, 304,385

Falco columbarius, 330 peregrinus, $82,330,376$

sparverius, 216,413

tinnunculus, 413

Falcon, Peregrine, 82,330,376

Felis domesticus, 208,268

Fern, Northern Bracken, 204

Royal, 184

Sensitive, 184

Feverfew, 44

Field-Naturalists' Club Awards for 2006, The Ottawa, 455
Field-Naturalists' Club 9 January 2007, Minutes of the 128th Annual Business Meeting of The Ottawa, 350

Finley, J.K. Offshore Flight of Buffleheads (Bucephala albeola) After Twilight in Winter: An Anti-Predation Tactic?, 375

Finley, J.K. The Punctual Bufflehead, Bucephala albeola: Autumn Arrivals in Shoal Harbour Sanctuary, Vancouver Island, in Relation to Freeze-up, 370

Fir, Balsam, 327,329,359

Douglas, 134

Subalpine, 156

Fireweed, 359

Fisher, 120,391,437

Flavopunctelia flaventior, 18

Fleabane, Canada, 184

Common, 184

Daisy, 184

Lesser, 184

Flint, P.L., 308

Flounder, Japanese, 35 Speckled, 37

Fly, Deer, 257

Flycatcher, Least, 244 Willow, 244

Fortin, C., M. Ouellet, I. Cartier, D. Banville, et C.B. Renaud. Biologie et situation de la Lamproie du Nord, IchthyoFox, 385 myzon fossor, au Québec, 402

Arctic, 73,120,308,322,330

Kit, 71

Red, 73,120,150,271,322,386

Swift, 71

Fox, Vulpes velox, Den Use Patterns in Northwestern Texas, Swift, 71

Foxtail, Green, 184

Yellow, 184

Fragaria vesca ssp. americana, 184

virginiana, 184

virginiana ssp. virginiana, 203

Fraser, E.E., 420

Fratercula arctica, 275,289

Fraxinus americana, 304 nigra, 385

Freedman, B., 265

Frey, B., C. Kempler, and D.L. Ehret. Micro-Propagation of White-top Aster, Sericocarpus rigidus, a Threatened Species from the Garry Oak Ecosystem in British Columbia, 40

Fritillary, Regal, 131

Frog, Green, 180

Frullania nisquallensis, 26

Fudge, D., B. Freedman, M. Crowell, T. Nette, and V. Power. Road-kill of Mammals in Nova Scotia, 265

Fulica americana, 50,245

Fumaria officinalis, 79

Gadus morhua, 285

Gadwall, 245

Gagnon, B., 67

Galinsoga quadriradiata, 184

Galium boreale, 203 circaezans var. circaezans, 203

obtusum, 184

trifidum ssp. trifidum, 184 
Gallinago delicata, 245

Gallinula chloropus, 326

Gallinule, Common, 326

Gannet, Northern, 275

Garbary, D.J. and A.E. Deveau. The Spindle Tree, Euonymus europaea L. (Celastraceae): A Newly Naturalized Shrub in Nova Scotia, 85

Garbary, D.J. and B.R. Taylor. Flowering During January in Antigonish County, Nova Scotia, 76

Gardensnail, White-lip, 434

Gavia pacifica, 325 stellata, 289

Gawn, M., Review by, 105

Gayfeather, Ontario, 203

Geese, Branta canadensis, Management Implications of Molt Migration by the Atlantic Flyway Resident Population of Canada, 313

Gentian, Bottle, 184

Gentiana andrewsii, 184

Geococcyx californianus, 216

Geothlypis trichas, 244

Geranium bicknellii, 184

Germander, 185

Geum aleppicum, 184 canadense, 184

Gibson, G.D., 168

Gibson, M., 214

Gilhen, J. and R. Strum. Arboreal Late Summer Courtship Behaviour of Maritime Garter Snake, Thamnophis sirtalis pallidulus, in Dartmouth, Nova Scotia, Canada, 210

Glaucomys sabrinus, 120,303,389 volans, 305

Glaucomys sabrinus, and North American Red Squirrels, Tamiasciurus hudsonicus, in a Secondary Hardwood Forest of Southern Ontario, Cavity Nest Materials of Northern Flying Squirrels, 303

Glyceria grandis, 166

Glyptemys insculpta, 178

Goat, Mountain, 121,156

Goatsbeard, 185

Showy, 185

Godwit, Marbled, 67

Godwit, Limosa fedoa, in Québec, First Confirmed Breeding of the Marbled, 67

Goldenrod, 44,182

Blue-stemmed, 184

Canada, 185,204

Early, 185,204

Grass-leaved, 184

Gray, 185,204

Late, 204

Old Field, 185

Rough-stemmed, 185

Tall, 185

Goldfinch, American, 245

Goodwin, C.E., Review by, 441

Goose, Canada, 245,313

Giant Canada, 316

Great Basin Canada, 316

Goosefoot, Maple-leaved, 184

Goshawk, Northern, 329,413

Goshawk, Accipiter gentilis, Exploits a Beagle Hound, Canis familiaris, as a "Beater" to Catch a Snowshoe Hare, Lepus americanus, Northern, 329

Götherström, A., 321
Grackle, Common, 243

Grama, Blue, 71

Side-oats, 71

Grape, River-bank, 204

Graptemys geographica, 178

Grass, Annual Blue, 184

Blue-eyed, 184

Canada Blue, 184

Hemlock Rosette, 203

Kentucky Blue, 183,203

Long Slim Leaf Rosette, 203

Manna, 166

Orchard, 184

Poverty Oat, 184

Poverty Wild Oat, 203

Reed Canary, 166

Slender Wheat, 184

Slim Leaf Rosette, 203

Starved Panic, 184

Starved Rosette, 203

Sweet, 68

Witch, 184

Woolly Panic, 184

Yellow Indian, 204

Grassbird, Little, 413

Grebe, Clark's, 50

Eared, 50

Horned, 50

Pied-billed, 245

Red-necked, 245

Slavonian, 325

Western, 50

Gregory, P.T., 142

Gromwell, 184

Groundhog, 271

Grouse, Ruffed, 329

Sharp-tailed, 245

Spruce, 329

Grus americana, 257

Guiasu, R.C., Review by, 109

Guillemot, 274

Gull, 274

Black, 275,288

Franklin's, 50

Glaucous, 308,328

Glaucous-winged, 325

Great Black-backed, 275,289,426,433

Herring, 275, 288,426,433

Ivory, 327

Ring-billed, 275,289

Gull, Larus marinus, eggs on Boot Island National Wildlife Area, Nova Scotia, Details of Eastern Coyote, Canis latrans, predation on Great Black-backed, 426

Gulls, Pagophila eburnea, in Inland Labrador, Winter Occurrences of Ivory, 327

Gulo gulo, 120,156,437

Gymnomitrion concinnatum, 26

obtusum, 26

pacificum, 26

Habenaria saccata, 24

Haliaeetus leucocephalus, 376

Halibut, Atlantic, 35

Halibut, Hippoglossus hippoglossus, from the Lower St. Lawrence Estuary, Quebec, A Rare Case of Completely Ambicoloured Atlantic, 35 
Halichoerus grypus, 392

Hall, W. Jr., 256

Haplopappus tenuisectus, 71

Hare, 385

Alaskan, 125

Arctic, 125

Snowshoe, 120,268,329,390,426

Hare, Lepus americanus, Northern Goshawk, Accipiter gentilis, Exploits a Beagle Hound, Canis familiaris, as a "Beater" to Catch a Snowshoe, 329

Harington, C.R. Giant Beaver, Castoroides ohioensis, Remains in Canada and an Overlooked Report from Ontario, 330

Harmsen, R., 182

Harrier, Northern, 245,330

Harriman, V.B., J.A. Pitt, and S. Larivière. Do Repugnant Scents Increase Survival of Ground Nests? A Test with Artificial and Natural Duck Nests, 150

Hawk, Broad-winged, 216

Cooper's, 216

Ferruginous, 245

Red-shouldered, 216

Rough-legged, 330

Swainson's, 245

Hawkweed, Orange, 184

Hedge-Mustard, 184

Heilhecker, E., R.P. Thiel, and W. Hall, Jr. Wolf, Canis lupus, Behavior in Areas of Frequent Human Activity, 256

Helianthus divaricatus, 203

Hemlock, Eastern, 304 Western, 24

Heracleum lanatum, 24

Herb, Hairy Willow, 184 Northern Willow, 166

Herbertus aduncus, 26 sakuraii, 26

Heron, Great Blue, 426 Little Blue, 54

Herrero, S., 155

Herring, 275

Atlantic, 433

Pacific, 325

Hieracium spp., 187

aurantiacum, 184

piloselloides, 184,202

Hierochloe odorata, 68

Hippoglossus hippoglossus, 35

Hippoglossus hippoglossus, from the Lower St. Lawrence Estuary, Quebec, A Rare Case of Completely Ambicoloured Atlantic Halibut, 35

Hirundo rustica, 244

Histrionicus histrionicus 82

Hong, W.S. The Hepatic Flora and Floristic Affinity of Hepatics Around Takakia Lake, Queen Charlotte Islands, British Columbia, 24

Hordeum jubatum, 165

Horehound, Cut-leaved Water, 184 Water, 166,184

Hornwort, 166

Horsetail, 359

Field, 184

Marsh, 166

Hound, Beagle, 329

Hound, Canis familiaris, as a "Beater" to Catch a Snowshoe Hare, Lepus americanus, Northern Goshawk, Accipiter gentilis, Exploits a Beagle, 329
Houston, C.S., Reviews by, 110,230,339

Howerter, D., 261

Hudson, R.J., 299

Huettmann, F., Review by, 442

Hydroprogne caspia, 289

Hygrobiella laxifolia, 26

Hypericum perforatum, 184,202

Hypogymnia physodes, 18

Ibis, White-faced, 46

Ibis, Plegadis chihi, Establishment in the Northern Prairie and Parkland Region of North America, Pattern and Potential Causes of White-faced, 46

Ichthyomyzon, 402

fossor, 402

unicuspis, 402

Ichthyomyzon fossor, au Québec, Biologie et situation de la Lamproie du Nord, 402

Icterus spurius, 245

Igl, L.D. and D.H. Johnson. Brown-headed Cowbird, Molothrus ater, Parasitism and Abundance in the Northern Great Plains, 239

Ishmael, W.E., 333

Isopod, Marine, 168

Isopod Cyathura polita in the Saint John River Estuary, New Brunswick: a Species at the Northern Extent of its Range, Life History of the Marine, 168

Jacques, C.N., W.E. Ishmael, T.R. Van Deelen, and R.E. Rolley. A Late Born White-tailed Deer, Odocoileus virginianus, Fawn in Southcentral Wisconsin, 333

Jandt, R.R., 379

John, R., Reviews by, 101,222,224,226,228,229,337,440,446

Johnson, D.H., 239

Joly, K., M.J. Cole, and R.R. Jandt. Diets of Overwintering Caribou, Rangifer tarandus, Track Decadal Changes in Arctic Tundra Vegetation, 379

Juncus dudleyi, 184

Juneberry, 89

Jung, T.S., 119,422

Jung, T.S., T.D. Pretzlaw, and D.W. Nagorsen. Northern Range Extension of the Pygmy Shrew, Sorex hoyi, in the Yukon, 94

Jungermannia hyaline, 26 obovata, 26 pumila, 26

Juniper, Creeping, 191

Juniperus horizontalis, 191

Kay, C.E., Review by, 220

Kay, C.E. Were Native People Keystone Predators? A Continuous-Time Analysis of Wildlife Observations Made by Lewis and Clark in 1804-1806, 1

Kempler, C., 40

Kennedy, A.C., 426

Kestrel, American, 216,413 European, 413

Killdeer, 130

Kilpatrick, C.W., 206

King, B., 201

Kingbird, Eastern, 243 Western, 243

Kittiwake, Black-legged, 275,288,327

Kiviat, E., 128

Knutsen, G.A., 46

Kurzia sylvatica, 26 
Kuzyk, G.W. and R.J. Hudson. Twenty-Four-Hour Activity Budgets of Mule Deer, Odocoileus hemionus, in the Aspen Parkland of Eastcentral Alberta, 299

Labrador, Moose, Alces alces, Winter Browse Use in Central, 359

Labrador, The Occurrence of Muskoxen, Ovibos moschatus in, 81

Labrador, Winter Occurrences of Ivory Gulls, Pagophila eburnea, in Inland, 327

Lacroix, D.L., 308

Lactuca canadensis, 184

Ladies' Tresses, Hooded, 185

Lampetra appendix, 403 planeri, 407

Lamproie argentée, 402

de l'Est, 403

de Planer, 407

du Nord, 402 marine, 406

Lamproie du Nord, Ichthyomyzon fossor, au Québec, Biologie et situation de la, 402

Lampsilis radiate, 93

Lanius ludovicianus, 244

Larivière, S., 150

Larivière, S., D. Howerter, and F. Messier. Influence of Gender and Den Type on Home Range Shape for Striped Skunks, Mephitis mephitis, in Saskatchewan, 261

Larix laricina, 67,256,385

Lark, Horned, 242

Larus argentatus, 275,289,426,433

delawarensis, 275,289

glaucescens, 325

hyperboreus, 308,328

marinus, 275,289,426,433

pipixcan, 50

Larus marinus, eggs on Boot Island National Wildlife Area, Nova Scotia, Details of Eastern Coyote, Canis latrans, predation on Great Black-backed Gull, 426

Lasionycteris noctivagans, 121

Lasiurus sp., 420 borealis, 124,208,386 cinereus, 124,208,386

Lauff, R.F., Review by, 219,340

Lechea intermedia var. intermedia, 203

Ledum groenlandicum, 81

Lemming, Nearctic Brown, 120

Nearctic Collared, 120

Northern Bog, 120

Ogilvie Mountains, 122

Ogilvie Mountains Collared, 120

Lemmus trimucronatus, 120

Lemna trisulca, 165

Leontodon autumnalis, 77

Leopardus pardalis, 265

Lepidium campestre, 78,184

Lepidozia filamentosa, 26 reptans, 26

Lepus americanus, 120,268,329,390,426

arcticus, 125

othus, 125

Lepus americanus, Northern Goshawk, Accipiter gentilis, Exploits a Beagle Hound, Canis familiaris, as a "Beater" to Catch a Snowshoe Hare, 329
Letharia, 303

Lettuce, Wild, 184

Lewis, C., Review by, 345

Lewis and Clark in 1804-1806, Were Native People Keystone Predators? A Continuous-Time Analysis of Wildlife Observations Made by, 1

Liatris cylindracea, 203

Lice, 431

Lilium philadelphicum var. philadelphicum, 203

Lily-of-the-valley, False, 203

Lily, Wood, 203 Yellow Water, 359

Lim, B.K., Review by, 105

Limberpine, 134

Limosa fedoa, 67

Limosa fedoa, in Québec, First Confirmed Breeding of the Marbled Godwit, 67

Liparis liliifolia, 297 loeselii, 297

Lithospermum officinale, 184

Lobelia inflata, 184

Longspur, Chestnut-collared, 242

Lontra canadensis, 120,325,388

Lontra canadensis, Capture of a Double-crested Cormorant, Phalacrocorax auritus, in British Columbia's Gulf Island Waters, A River Otter's, 325

Loon, Pacific, 325

Red-throated, 288

Loosestrife, Yellow, 184

Lophozia guttulata, 26 incisa, 26

longiflora, 26

opacifolia, 26

sudetica, 26

ventricosa, 26

ventricosa var. longiflora, 26 wenzelii, 26

Lutra lutra, 394

Lycopus americanus, 184 asper, 166 uniflorus, 184

Lynx canadensis, 120,437 lynx, 388 rufus, 437

Lynx, 385 Canada, 120,388,437

Lyogyrus granum, 92 pupoidea, 92

Lyogyrus granum (Mollusca), Widespread in the Hampton Marsh, New Brunswick, A Freshwater Hydrobiid, cf. the Squat Duskysnail, 92

Lysichiton americanus, 421

Lysimachia terrestris, 184

MacCulloch, R.D., Review by, 104

MacKinnon, C.M., A.C. Kennedy, and D.W. Colpitts. Details of Eastern Coyote, Canis latrans, predation on Great Black-backed Gull, Larus marinus, eggs on Boot Island National Wildlife Area, Nova Scotia, 426

Macropus rufogriseus, 268

Madder, Wild, 184

Magpie, Black-billed, 150

Maianthemum canadense, 203 stellatum, 203

Maillet, R.H., 92 
Malaclemys terrapin, 178

Malcolm, J.R., 303

Malecki, R.A., 313

Mallard, 130,245

Mallotus villosus, 285

Mammut americanum, 332

Maple, 420

$$
\begin{aligned}
& \text { Mountain, } 359 \\
& \text { Red, 385 } \\
& \text { Sugar, 304,385 }
\end{aligned}
$$

Manitoba and Northwestern Ontario, Recent Distribution Records of the Little Brown Bat, Myotis lucifugus, in, 57

Manitoba, Growth of White Spruce, Picea glauca, Seedlings in Relation to Microenvironmental Conditions in a Forest-Prairie Ecotone of Southwestern, 191

Marigold, 44

Marine Turtle Newsletter, 117,233,349,453

Marmot, Alaska, 125 Hoary, 120

Marmota broweri, 125 caligata, 120 monax, 120,271

Marr, K.L., 421

Marsupella alpina, 26 boeckii, 26 emarginata, 27 emarginata var. aquatica, 26

Martel, A.L., 92

Marten, 385 American, 120,387,422 Pine, 394

Marten, Martes americana, Apparent Predation of an American Water Shrew, Sorex palustris, by an American, 422

Martes americana, 120,387,422 martes, 394 pennanti, 120,391,437

Martes americana, Apparent Predation of an American Water Shrew, Sorex palustris, by an American Marten, 422

Martin, R.E., 46

Massachusetts, Movements of Transient Coyotes, Canis latrans, in Urbanized Eastern, 364

Mastodon, American, 332

Matricaria maritime, 77

matricarioides, 77

McAlpine, D.F., 208

McAlpine, D.F., R.H. Maillet, A.J. Albert, L.M. Crossman, R.R. Smith, and A.L. Martel. A Freshwater Hydrobiid, cf. the Squat Duskysnail, Lyogyrus granum (Mollusca), Widespread in the Hampton Marsh, New Brunswick, 92

McGee, B.K., W.B. Ballard, and K.L. Nicholson. Swift Fox, Vulpes velox, Den Use Patterns in Northwestern Texas, 71

McNicholl, M., Reviews by, 223,227

Meadowlark, Eastern, 252

Western, 242

Medicago lupulina, 184 sativa ssp. falcata, 184 sativa ssp. sativa, 184

Medick, 184

$$
\text { Black, } 184
$$

Megalurus gramineus, 413

Meijer, T., 321
Melampyrum lineare var. lineare, 203

Melanelia albertana, 18

Meleagris gallopavo, 245

Meles meles, 265

Melilotus alba, 165,184 officinalis, 184

Melospiza georgiana, 244 melodia, 244

Mentha arvensis ssp. borealis, 184

Menyanthes trifoliata, 421

Mephitis mephitis, 125,150,261,266,392

Mephitis mephitis, in Saskatchewan, Influence of Gender and Den Type on Home Range Shape for Striped Skunks, 261

Mercer, S.C., G.D. Gibson, and M.J. Dadswell. Life History of the Marine Isopod Cyathura polita in the Saint John River Estuary, New Brunswick: a Species at the Northern Extent of its Range, 168

Merlin, 330

Messier, F., 261

Microtus canicaudus, 416 chrotorrhinus, 206

longicaudus, 120

miurus, 120

oeconomus, 120

pennsylvanicus, 120,390,416

xanthognathus, 120

Milfoil, 164 Water, 166

Milkweed, 187

Butterfly, 203

Common, 184,Common, 203

Poke, 203

Milkwort, Racemed, 203

Miller, R., 35

Mimus polyglottos, 244

Mink, 271,385 American, 120,150,388,422

Mint, Wild, 184

Mitchell, M.S., 412

Mitella pentandra, 24

Mitrow, G., 89

Mochnacz, N.J., 437

Mockingbird, Northern, 244

Moerckia blyttii, 27

Mole, 385

$$
\text { Star-nosed, } 206
$$

Mole (Condylura cristata) in Northeastern Vermont, A High Elevation Record of the Star-nosed, 206

Molothrus ater, 239

Molothrus ater, Parasitism and Abundance in the Northern Great Plains, Brown-headed Cowbird, 239

Monarda fistulosa ssp. fistulosa var. fistulosa, 203

Monson, K.E., 57

Moose, 121,156,265,359,385,416

Moose, Alces alces, Winter Browse Use in Central Labrador, 359

Morneau, F., B. Gagnon, and S. Whiskeychan. First Confirmed Breeding of the Marbled Godwit, Limosa fedoa, in Québec, 67

Morus bassanus, 275

Mosquito, 257

Moss, Feather, 360

Mouse, 385

Deer, 206,390 
House, 125,392

Meadow Jumping, 120,390

Western Jumping, 120

Woodland Jumping, 271,390

Moxostoma sp., 29

anisurum, 29

carinatum, 29

duquesnei, 29

macrolepidotum, 29

valenciennesi, 29

Moxostoma, Species, Comparison of Scales, Pectoral Fin Rays and Opercles for Age Estimation of Ontario Redhorse, 29

Muhlenbergia mexicana, 183

Mullein, Common, 185

Murre, Common, 275,288

Mus domesticus, 392

musculus, 125

Muskox, 81,121

Muskoxen, Ovibos moschatus in Labrador, The Occurrence of, 81

Muskrat, 122,271,385

Common, 120

Mustard, Hairy-pod Hedge, 426

Mustela erminea, 120,388

nivalis, 120

vison, $123,271,388$

Mylia taylorii, 27

Myodes, 119

gapperi, 120,206

rutilus, 120

Myotis, 119,209,420

evotis, 124

formosus, 420

keenii, 124

lucifugus, 57,120,208,386,420

septentrionalis, 120,208,386

volans, 124

Myotis, Keen's, 124

Little Brown, 120

Long-eared, 125

Long-legged, 125

Northern, 120

Myotis lucifugus, in Manitoba and Northwestern Ontario, Recent Distribution Records of the Little Brown Bat, 57

Myotis lucifugus, Observation of Foliage-roosting in the Little Brown Bat, 420

Myotis septentrionalis (Chiroptera: Vespertilionidae), on Prince Edward Island: First Records of Occurrence and Over-Wintering, Northern Long-eared Bat, 208

Myriophyllum sibiricum, 164

Nagorsen, D.W., 94

Napaeozapus insignis, 390

Nardia compressa, 27

geoscyphus, 27

japonica, 27

scalaris, 27

Nelson, K.J., 142

Neotamias minimus, 122

Neotoma cinerea, 120

fuscipes, 305

Neovison vison, $120,150,422$

Nette, T., 265
Neville, J.T. Northern Goshawk, Accipiter gentilis, Exploits a Beagle Hound, Canis familiaris, as a "Beater" to Catch a Snowshoe Hare, Lepus americanus, 329

New Brunswick, A Freshwater Hydrobiid, cf. the Squat Duskysnail, Lyogyrus granum (Mollusca), Widespread in the Hampton Marsh, 92

New Brunswick: a Species at the Northern Extent of its Range, Life History of the Marine Isopod Cyathura polita in the Saint John River Estuary, 168

New Hampshire, Post-Emergence Movements and Overwintering of Snapping Turtle, Chelydra serpentina, Hatchlings in New York and, 178

New Jersey, State Records and Habitat of Clam Shrimp, Caenestheriella gynecia (Crustacea: Conchostraca), in New York and, 128

New York and New Hampshire, Post-Emergence Movements and Overwintering of Snapping Turtle, Chelydra serpentina, Hatchlings in, 178

New York and New Jersey, State Records and Habitat of Clam Shrimp, Caenestheriella gynecia (Crustacea: Conchostraca), in, 128

Newbury, T.L., N.P.P. Simon, and T.E. Chubbs. Moose, Alces alces, Winter Browse Use in Central Labrador, 359

Nicholson, K.L., 71

Night-Heron, Black-crowned, 50

Nighthawk, Common, 245

Norris, R.W. and C.W. Kilpatrick. A High Elevation Record of the Star-nosed Mole (Condylura cristata) in Northeastern Vermont, 206

North America, Pattern and Potential Causes of White-faced Ibis, Plegadis chihi, Establishment in the Northern Prairie and Parkland Region of, 46

Northwest Territories, Bull Trout, Salvelinus confluentus, and North American Porcupine, Erethizon dorsatum, Interaction in the Mackenzie Mountains, 437

Nova Scotia, Canada, Arboreal Late Summer Courtship Behaviour of Maritime Garter Snake, Thamnophis sirtalis pallidulus, in Dartmouth, 210

Nova Scotia, Details of Eastern Coyote, Canis latrans, predation on Great Black-backed Gull, Larus marinus, eggs on Boot Island National Wildlife Area, 426

Nova Scotia, Flowering During January in Antigonish County, 76

Nova Scotia, Road-kill of Mammals in, 265

Nova Scotia, The Spindle Tree, Euonymus europaea L. (Celastraceae): A Newly Naturalized Shrub in, 85

Nuphar microphyllum, 359

Nuthatch, Red-breasted, 428

Nuthatch, Sitta canadensis, Dies Stuck in Resin at Entrance to a Nest-box, Red-breasted, 428

Nycticorax nycticorax, 50

O’Neill, J., Review by, 448

Oak, Black, 202

Bur, 150

Garry, 40

Hill's, 257

Northern Red, 204

Northern White, 204

Red, 202

Oak Ecosystem in British Columbia, Micro-Propagation of White-top, Micro-Propagation of White-top Aster, Sericocarpus rigidus, a Threatened Species from the Garry, 40

Oceanodroma furcata, 325 
leucorhoa, 275,289,325

Ocelot, 265

Ochotona collaris, 120

Ochrolechia, 21 arborea, 18

Octopus, Giant Pacific, 423

Octopus, Enteroctopus dofleini, Attacks on Divers, Giant Pacific, 423

Odobenus rosmarus, 389

Odocoileus spp., 151,413 hemionus, 121,271,299

hemionus hemionus, 156

virginianus, 121,139,257,265,302,333,391,412,426, 430

Odocoileus hemionus, in the Aspen Parkland of Eastcentral Alberta, Twenty-Four-Hour Activity Budgets of Mule Deer, 299

Odocoileus virginianus, Fawn in Southcentral Wisconsin, A Late Born White-tailed Deer, 333

Odocoileus virginianus, Seasonal Variation in Sex Ratios Provides Developmental Advantages in White-tailed Deer, 412

Oenanthe sarmentosa, 421

Oenothera biennis, 166 perennis, 184

Omble de fontaine, 406

Ondatra zibethicus, 120,271,390

Onoclea sensibilis, 184

Ontario, Cavity Nest Materials of Northern Flying Squirrels, Glaucomys sabrinus, and North American Red Squirrels, Tamiasciurus hudsonicus, in a Secondary Hardwood Forest of Southern, 303

Ontario, Giant Beaver, Castoroides ohioensis, Remains in Canada and an Overlooked Report from, 330

Ontario, Recent Distribution Records of the Little Brown Bat, Myotis lucifugus, in Manitoba and Northwestern, 57

Ontario Redhorse, Moxostoma, Species, Comparison of Scales, Pectoral Fin Rays and Opercles for Age Estimation of, 29

Ontario, Serviceberry, Amelanchier intermedia, Escaped from Cultivation in Eastern, 89

Ontario: the Herbs, Notes on Succession in Old Fields in Southeastern, 182

Opossum, 212 Virginia, 416

Opossums, Didelphis virginiana, between the United States and Canada, Human-assisted Movements of Raccoons, Procyon lotor, and, 212

Orchid, Sparrow's-egg Lady's-slipper, 295

Orchid, Cypripedium passerinum, in Southwestern Yukon, Discovery of a Possibly Relict Outbreeding Morphotype of Sparrow's-egg Lady's-slipper, 295

Oreamnos americanus, 121,156

Oriole, Orchard, 245

Osmunda regalis, 184

Oswego-tea, 203

Otter, 385

American, 392

European, 394

North American River, 120

River, 325,376,388

Otter's, Lontra canadensis, Capture of a Double-crested Cormorant, Phalacrocorax auritus, in British Columbia's Gulf Island Waters, A River, 325
Ouellet, M., 402

Ovenbird, 244

Ovibos moschatus, 81,121

Ovibos moschatus in Labrador, The Occurrence of Muskoxen, 81

Ovis canadensis, 416 dalli, 121

Owl, Great Horned, 437 Short-eared, 245

Oxalis stricta, 184

Oxypolis occidentalis, 421 rigidior, 421

Oxypolis occidentalis, A New Native Vascular Plant Species for the Queen Charlotte Islands, British Columbia, Cowbane, 421

Oxyura jamaicensis, 50

Ozoga, J.J., 412

Pagophila eburnea, 327

Pagophila eburnea, in Inland Labrador, Winter Occurrences of Ivory Gulls, 327

Pagophilus groenlandicus, 327

Panicum acuminatum, 183 capillare, 184 depauperatum, 184 lanuginosum, 187

Panther, Florida, 265

Paquet, P., 155

Paralichthys olivaceus, 37 woolmani, 37

Parelaphostrongylus tenuis, 63

Parker, G.H. and C.G. Blomme. Fish-line Entanglement of Nesting Mourning Dove, Zenaida macroura, 436

Parmelia sulcata, 18

Parmeliopsis ambigua, 18

Parnis, E., Review by, 230

Parsnip, Water, 166

Partridge, Ice, 327

Passer domesticus, 428

Passerculus sandwichensis, 244

Patterson, J.E.H., S.J. Patterson, and J.R. Malcolm. Cavity Nest Materials of Northern Flying Squirrels, Glaucomys sabrinus, and North American Red Squirrels, Tamiasciurus hudsonicus, in a Secondary Hardwood Forest of Southern Ontario, 303

Patterson, S.J., 303

Pellia endiviifolia, 27 epiphylla, 27 neesiana, 27

Peltigera spp., 380

Pennycress, Field, 185

Penstemon hirsutus, 203

Peppergrass, Field, 184

Peromyscus, 119

keeni, 120

maniculatus, 120,206,390

maniculatus algidus, 122

Petromyzon marinus, 406

Phaeophyscia orbicularis, 18

Phalacrocorax auritus, 275,289,325,426 carbo, 275,289 pelagicus, 325

Phalacrocorax auritus, in British Columbia's Gulf Island Waters, A River Otter's, Lontra canadensis, Capture of a Double-crested Cormorant, 325 
Phalaris arundinacea, 166

Phalarope, Wilson's, 245

Phalaropus tricolor, 245

Phasianus colchicus, 245

Pheasant, Ring-necked, 245

Phenacomys ungava, 120

Phillips, F.R., 96,327

Phleum pratense, 182

Phoca groenlandica, 389 vitulina, 376,388

Phoebe, Say's, 244

Phragmites australis, 129

Physalis heterophylla, 184,203

Physcia adscendens, 18 aipolia, 18

Pica pica, 150

Picea, 297

engelmannii, 134,156

glauca, 191,304,327,426

glauca $\times$ engelmannii, 156

mariana, 256,304,327,329,360,385

pungens, 436

rubens, 304

sitchensis, 24

Picea glauca, Seedlings in Relation to Microenvironmental Conditions in a Forest-Prairie Ecotone of Southwest-

Pig, 413

ern Manitoba, Growth of White Spruce, 191

Pika, Collared, 120

Pillar, Glossy, 434

Pine, 256,413,428

Eastern White, 203,304

Lodgepole, 134

Red, 202,258

Scots, 201

White, 202

White-barked, 134

Pine, Pinus sylvestris, Natural Recolonization of Cultivated Land by Native Prairie Plants and its Enhancement by Removal of Scots, 201

Pink, Deptford, 184

Pintail, Northern, 245

Pinus sp., 413,428

albicaulis, 134

banksiana, 256

contorta, 134

flexilis, 134,

resinosa, 202,256

strobus, 202,256,304

sylvestris, 201

Pinus sylvestris, Natural Recolonization of Cultivated Land by Native Prairie Plants and its Enhancement by Removal of Scots Pine, 201

Pinweed, Round Fruit, 203

Pipilo maculatus, 244

Pipistrelle, Eastern, 420

Pipistrellus subflavus, 420

Pipit, Sprague's, 244

Pitt, J.A., 150

Plagiochila porelloides, 27 semidecurrens, 28

Plantago lanceolata, 184 major, 184 rugelii, 184

Plantain, Broad-leaved Water, 166 Common, 184
Narrow-leaved, 184

Rugel's, 184

Plectrophenax nivalis, 310

Plegadis chihi, 46

Plegadis chihi, Establishment in the Northern Prairie and Parkland Region of North America, Pattern and Potential Causes of White-faced Ibis, 46

Pleurocladula albescens, 26

Pleurozia purpurea, 26

Pleurozium schreberi, 360

Poa alpina, 134

аппиа, 183

capillare, 183

compressa, 183,202

pratensis, 183

pratensis ssp. pratensis, 184,203

Podiceps auritus, 50,325

grisegena, 245

nigricollis, 50

Podilymbus podiceps, 245

Poecile atricapillus, 428 rufescens, 428

Poison Ivy, 202

Ivy, Western, 204

Polygala polygama, 203

Polygonatum biflorum var. commutatum, 203

Polygonum arenastrum, 79

convolvulus, 184

persicaria, 184

sagittatum, 184

Pondweed, 164

Clasping Leaf, 166

Flat-stemmed, 166

Horned, 166

Large-sheath, 166

Leafy, 166

Narrow-leaved, 166

Sago, 166

Pooecetes gramineus, 244

Poplar, Balsam, 18

Populus, 297

balsamifera, 18

grandidentata, 256,304

tremuloides, 18,150,191,256,261,304,327

Porcupine, 266

American, 391

North American, 120,437

Porcupine, Erethizon dorsatum, Interaction in the Mackenzie Mountains, Northwest Territories, Bull Trout, Salvelinus confluentus, and North American, 437

Porzana carolina, 245,377

Potamogeton, 164

filiformis, 166

pectinatus, 165

perfoliatus, 93

richardsonii, 165

vaginatus, 166

zosteriformis, 166

Potentilla argentea, 184

arguta ssp. arguta, 203

canadensis, 184

inclinata, 184

norvegica, 184,202

recta, 184

simplex, 203 
Powell, T., T.S. Jung and K.J. Clyde. Apparent Predation of an American Water Shrew, Sorex palustris, by an American Marten, Martes americana, 422

Power, V., 265

Pretzlaw, T.D., 94

Price, M.H.H. and C.E. Aries. A River Otter's, Lontra canadensis, Capture of a Double-crested Cormorant, Phalacrocorax auritus, in British Columbia's Gulf Island Waters, 325

Primrose, Yellow-Evening, 166

Prince Edward Island, An Analysis of the Historical Records for the Native Mammalian Fauna of, 384

Prince Edward Island: First Records of Occurrence and Over-Wintering, Northern Long-eared Bat, Myotis septentrionalis (Chiroptera: Vespertilionidae), on, 208

Procastoroides, 331

Procyon lotor, 130,150,212,266,391

Procyon lotor, and Opossums, Didelphis virginiana, between the United States and Canada, Human-assisted Movements of Raccoons, 212

Prunella vulgaris, 187 vulgaris ssp. lanceolata, 187

Prunus, 86 vulgaris ssp. vulgaris, 184

pennsylvanica, 359

pumila var. susquehanae, 203

serotina, 203

virginiana var. virginiana, 203

Pseudoroegneria spicata, 134

Pseudotsuga menziesii, 134

Pteridium aquilinum var. latiusculum, 204

Ptilidium californicum, 27

Puffin, Atlantic, 275,288

Puma concolor, 120,437

concolor coryi, 265

Purse, Shepherd's, 184

Pusa hispida, 98

Pussytoes, Field, 184

Parlin's, 203

Plantain-leaved, 184

Small, 203

Quebec, A Rare Case of Completely Ambicoloured Atlantic Halibut, Hippoglossus hippoglossus, from the Lower St. Lawrence Estuary, 35

Québec, Biologie et situation de la Lamproie du Nord, Ichthyomyzon fossor, au, 402

Québec, First Confirmed Breeding of the Marbled Godwit, Limosa fedoa, in, 67

Québec, 2002, Third Census of Seabird Populations of the Gaspé Peninsula, 274

Quercus alba, 204,256

borealis, 256

ellipsoidalis, 257

macrocarpa, 150

relutina, 202

rubra, 202

velutina, 204,256

Quickweed, 184

Quiscalus quiscula, 245

Rabbit, 385

Raccoon, 130,150,212,266,391

Raccoons, Procyon lotor, and Opossums, Didelphis virginiana, between the United States and Canada, Human-assisted Movements of, 212
Radish, Wild, 426

Radula auriculata, 27 obtusiloba ssp. polyclada, 27

Ragweed, 187 Common, 184

Rail, J.-F., 274

Rail, J.-F. and R. Cotter. Sixteenth Census of Seabird Populations in the Sanctuaries of the North Shore of the Gulf of St. Lawrence, 2005, 287

Rana clamitans, 180

Rangifer tarandus, 82,121,327,362,379,389 tarandus caribou, 96,155

Rangifer tarandus caribou, Herd in Westcentral British Columbia, 1997-2005, Translocation and Recovery Efforts for the Telkwa Caribou, 155

Rangifer tarandus, Track Decadal Changes in Arctic Tundra Vegetation, Diets of Overwintering Caribou, 379

Ranunculus abortivus, 184

acris, 184

aquatilis, 166

rhomboideus, 204

Raphanus raphanistrum, 78,426

Raspberry, Common Red, 204

Rat, Brown, 392 Dusky-footed Wood, 305

Rattus norvegicus, 392

Raven, Common, 150,427

Razorbill, 274,284,288

Recurvirostra americana, 245

Reed, J.A., D.L. Lacroix, and P.L. Flint. Depredation of Common Eider, Somateria mollissima, Nests on a Central Beaufort Sea Barrier Island: A Case Where No One Wins, 308

Redhead, 245

Redhorse, Black, 29

Greater, 29

Ontario, 29

River, 29

Shorthead, 29

Silver, 29

Redhorse, Moxostoma, Species, Comparison of Scales, Pectoral Fin Rays and Opercles for Age Estimation of Ontario, 29

Redtop, 184

Reed, Common, 129

Reid, S.M. Comparison of Scales, Pectoral Fin Rays and Opercles for Age Estimation of Ontario Redhorse, Moxostoma, Species, 29

Remarchu, K., 164

Renaud, C.B., 402

Rhododendron sp., 210

Rhododendron, 210

Rhus radicans, 202 typhina, 204

Rhynchospora knieskernii, 131

Riccardia latifrons, 27 palmata, 27

Rissa tridactyla, 275,289,327

Roadrunner, Greater, 216

Robin, American, 216,244

Robin, Turdus migratorius, and a Five-lined Skink, Eumeces fasciatus, Predator-Prey Interaction Between an American, 216

Rockcress, 203

Hairy, 203

Holboell's, 203 
Rolley, R.E., 333

Rosa acicularis ssp. acicularis, 204 blanda var. blanda, 204

Rosatte, R. Immobilization of Elk, Cervus elaphus, with Telezol and Xylazine and Reversal with Tolazine or Yohimbine, 62

Rosatte, R., A. Silver, M. Gibson, B. Chisholm, and N. Cool. Correlation Between Age Estimates for Elk, Cervus Elaphus, Using Tooth Wear/Eruption Patterns and Counts of Annuli in Tooth Cementum, 214

Rosatte, R., D. Donovan, M. Allan, L. Bruce, and C. Davies. Human-assisted Movements of Raccoons, Procyon lotor, and Opossums, Didelphis virginiana, between the United States and Canada, 212

Rose, Prickly, 204

Smooth, 204

Rubus spp., 204

idaeus ssp. idaeus, 204

Rudbeckia hirta, 184

hirta var. hirta, 204

Rumex acetosella, 187,202

acetosella ssp. acetosella, 184

crispus, 184

Rush, Dudley's, 184

Knieskern's Beaked, 131

Needle-Spike, 166

Scouring, 184

Rye, Slender Wild, 203

Saalfeld, S.T., S.S. Ditchkoff, J.J. Ozoga, and M.S. Mitchell. Seasonal Variation in Sex Ratios Provides Developmental Advantages in White-tailed Deer, Odocoileus virginianus, 412

Safflower, 44

Sagebrush, 134

Salix sp., 81,359

exigua, 166

humilis var. humilis, 204

Salvelinus confluentus, 437 fontinalis, 406

Salvelinus confluentus, and North American Porcupine, Erethizon dorsatum, Interaction in the Mackenzie Mountains, Northwest Territories, Bull Trout, 437

Sambucus racemosa, 426

Sander-Regier, R., Reviews by, 338,341,346,447,449

Sandlance, 285

Pacific, 325

Sandpiper, Common, 413 Upland, 242

Sanicula marilandica, 184

Saskatchewan, Canada, Before and After the Application of the Herbicide Magnacide, An Inventory of the Aquatic and Subaquatic Plants in SASKWater Canals in Central, 164

Saskatchewan, Influence of Gender and Den Type on Home Range Shape for Striped Skunks, Mephitis mephitis, in, 261

Saskatoon, 89

Saussurea obvallata, 44

Saxifraga punctata, 24

Sayornis saya, 244

Scapania americana, 27

bolanderi, 27

paludosa, 27

uliginosa var. oakesii, 27 undulate, 27

undulate var. oakesii, 27

Scaup, Lesser, 245

Schizachyrium scoparium var. scoparium, 204

Schmidt, R.E. and E. Kiviat. State Records and Habitat of Clam Shrimp, Caenestheriella gynecia (Crustacea: Conchostraca), in New York and New Jersey, 128

Schoenoplectus acutus, 54,166 tabernaemontani, 166

Schultz, R.N., A.P. Wydeven, L.S. Winn, and S.A. Buller. Attempt to Cross-Foster Gray Wolf, Canis lupus, Pups into Another Wolf Pack, 430

Scirpus microcarpus, 166

Sciurus carolinensis, 304,392

Scribner, K., 313

Sea cow, 385

Seal, 385

Grey, 392

Harbour, 376,388

Harp, 327,389

Hooded, 392

Ringed, 98

Seburn, D., Reviews by, 106,343

Sedge, 134,184,359

Back's, 203

Beaked, 166

Common, 184

Dry-spike, 203

Filiform, 184

Fox, 184

Graceful, 203

Granular, 184

Great Plains Flat, 203

Houghton's Flat, 203

Muhlenberg's, 203

Normal, 184

Pennsylvania, 203

Richardson's, 203

Slender, 184

Troublesome, 203

Seiurus aurocapillus, 244

Self-Heal, 184

Senecio triangularis, 24,421 vulgaris, 77

Sericocarpus rigidus, 40

Sericocarpus rigidus, a Threatened Species from the Garry Oak Ecosystem in British Columbia, Micro-Propagation of White-top, Aster, 40

Serviceberry, 89

Allegheny, 203

Downy, 203

Running, 203

Serviceberry, Amelanchier intermedia, Escaped from Cultivation in Eastern Ontario, 89

Setaria pumila, 183 viridis, 183

Shadbush, 89

Shaffer, J.A., G.A. Knutsen, R.E. Martin, and J.S. Brice. Pattern and Potential Causes of White-faced Ibis, Plegadis chihi, Establishment in the Northern Prairie and Parkland Region of North America, 46

Shapiro, B., 321

Sharpe, S., 155

Sheaffer, S.E., R.A. Malecki, B.L. Swift, J. Dunn, and K. Scribner. Management Implications of Molt Migra- 
tion by the Atlantic Flyway Resident Population of Canada Geese, Branta canadensis, 313

Sheep, Bighorn, 416

Dall's, 121

Shepherdia canadensis, 204

Shimek, R., 423

Shoveler, Northern, 245

Shrew, 385

Alaskan Tiny, 94,124

American Pygmy, 120

American Water, 94,120,422

Arctic, 120

Barren Ground, 120

Cinereus, 120

Dusky, 120

Masked, 386

Northern Short-tailed, 386

Pygmy, 94,121,386

Short-tailed, 206

Smoky, 206,386

Tundra, 94,120

Water, 386

Shrew, Sorex hoyi, in the Yukon, Northern Range Extension of the Pygmy, 94

Shrew, Sorex palustris, by an American Marten, Martes americana, Apparent Predation of an American Water Shrew, 422

Shrike, Loggerhead, 244

Shrimp, Clam, 128

Shrimp, Caenestheriella gynecia (Crustacea: Conchostraca), in New York and New Jersey, State Records and Habitat of, 128

Silene vulgaris, 184

Silver, A., 214

Simon, N.P.P., 359

Simon 1973-2006, A Tribute to Neal Philip Perry, 96

Sisymbrium officinale, 184,426

Sisyrinchium angustifolium, 184

Sitta canadensis, 428

Sitta canadensis, Dies Stuck in Resin at Entrance to a Nestbox, Red-breasted Nuthatch, 428

Sium suave, 166

Skink, Five-lined, 216

Skink, Eumeces fasciatus, Predator-Prey Interaction Between an American Robin, Turdus migratorius, and a Fivelined, 216

Skipper, European, 182

Skunk, Striped, 125,150,261,266,392

Skunks, Mephitis mephitis, in Saskatchewan, Influence of Gender and Den Type on Home Range Shape for Striped, 261

Slough, B.G. and T.S. Jung. Diversity and Distribution of the Terrestrial Mammals of the Yukon Territory: A Review, 119

Smilacina stellata, 203

Smith, C.M., Reviews by, 101,103

Smith, R.R., 92

Smith, T.G., Review by, 107

Snail, Ordinary Spire, 92

Snake, Eastern Garter, 210

Maritime Garter, 210

Red-sided Garter, 210

Sharp-tailed, 124

Snake, Contia tenuis, in Southwestern British Columbia, The Rumsfeld Paradigm: Knowns and Unknowns in
Characterizing Habitats Used by the Endangered Sharp-tailed, 142

Snake, Thamnophis sirtalis pallidulus, in Dartmouth, Nova Scotia, Canada, Arboreal Late Summer Courtship Behaviour of Maritime Garter, 210

Snakeroot, Black, 184

White, 184

Snipe, Wilson's, 245

Sobey, D.G. An Analysis of the Historical Records for the Native Mammalian Fauna of Prince Edward Island, 384

Solidago ssp., 182

caesia, 184

canadensis, 44,182

canadensis var. canadensis, 204

gigantea, 44,185,204

graminifolia, 44

juncea, 185,204

nemoralis, 185,204

rugosa, 185

virgaurea, 44

Solomon's Seal, King, 203

Starry False, 203

Somateria dresseri, 275 mollissima, 275,289,308

mollissima v-nigra, 308

Somateria mollissima, Nests on a Central Beaufort Sea Barrier Island: A Case Where No One Wins, Depredation of Common Eider, 308

Sonchus asper ssp. asper, 185

Sora, 245,377

Sorbus americana, 359

Sorex arcticus, 120

cinereus, $120,386,422$

fumeus, 206,386

hoyi, $94,120,386,422$

monticolus, 120,422

palustris, 94,120,386,422

tundrensis, 94,120

ugyunak, 120

yukonicus, 94,124

Sorex hoyi, in the Yukon, Northern Range Extension of the Pygmy Shrew, 94

Sorex palustris, by an American Marten, Martes americana, Apparent Predation of an American Water Shrew, 422

Sorghastrum nutans, 204

Sorrel, Sheep, 184 Yellow Wood, 184

Sow-thistle, Spiny, 185

Sparganium sp., 166

Sparrow, Baird's, 244

Brewer's, 244

Chipping, 244

Clay-colored, 242

Field, 244

Grasshopper, 242,244

Henslow's, 244

House, 428

Lark, 244

Le Conte's, 244

Savannah, 242

Song, 244

Swamp, 244

Vesper, 244 
Speedwell, Thyme-leaved, 185

Spergula arvensis, 78

Spergularia rubra, 78

Spermophilus parryii, 120

Speyeria idalia, 131

Sphagnum, 256

Spike-Rush, Common, 166

Pale, 166

Spindle Tree, Euonymus europaea L. (Celastraceae): A Newly Naturalized Shrub in Nova Scotia, The, 85

Spiranthes ovalis var. erostellata, 297 ovalis var. ovalis, 297 romanzoffiana, 185

Spiza americana, 245

Spizella brewerii, 244

pallida, 244

passerina, 244

pusilla, 244

Spruce, 297,304

Black, 327,329,360,385,436

Engelmann, 134,156

Hybrid White, 156

Sitka, 24

White, 191,327,426

Spruce, Picea glauca, Seedlings in Relation to Microenvironmental Conditions in a Forest-Prairie Ecotone of Southwestern Manitoba, Growth of White, 191

Squirrel, 385

American Red, 389

Arctic Ground, 120

Eastern Grey, 304,392

Flying, 385

North American Red, 303

Northern Flying, 120,303,389

Red, 120,268,271,329,385

Southern Flying, 305

Squirrels, Glaucomys sabrinus, and North American Red Squirrels, Tamiasciurus hudsonicus, in a Secondary Hardwood Forest of Southern Ontario, Cavity Nest Materials of Northern Flying, 303

Squirrels, Tamiasciurus hudsonicus, in a Secondary Hardwood Forest of Southern Ontario, Cavity Nest Materials of Northern Flying Squirrels, Glaucomys sabrinus, and North American Red, 303

St. John's Wort, Common, 184

Starling, Spotted, 413

Stellaria borealis ssp. borealis, 185

graminea, 185

media, 78,185

Sterna aleutica, 325

forsteri, 50

hirundo, 275,289,433

paradisaea, 289,433

Sternotherus odoratus, 178

Stitchwort, 185

Northern, 185

Storm-Petrel, Leach's, 275,289,325

Strauss, C.F. Red-breasted Nuthatch, Sitta canadensis, Dies Stuck in Resin at Entrance to a Nest-box, 428

Strawberry, Virginia, 203

Wild, 184

Wood, 184

Strawberry-Bush, Running, 85

Stronen, A.V., P. Paquet, S. Herrero, S. Sharpe, and N. Waters. Translocation and Recovery Efforts for the Telkwa
Caribou, Rangifer tarandus caribou, Herd in Westcentral British Columbia, 1997 2005, 155

Strum, R., 210

Sturgeon, Shortnose, 176

Sturnella magna, 252 neglecta, 245

Sturnus unicolor, 413

Sundrops, 184

Sunflower, Woodland, 203

Sus scrofa, 413

Susan, Black-eyed, 184,204

Swallow, Barn, 244 Violet-green, 428

Sweet-fern, 203

Swift, B.L., 313

Symphiotrichum lanceolatum ssp. hesperium, 166 ericoides var. ericoides, 204

novae-angliae, 204

oolentangiense var. oolentangiense, 204

Synaptomys borealis, 120

Syringa vulgaris, 76

Tachycineta thalassina, 428

Tagetes erecta, 44

Takakia lepidozioides, 24

Tamarack, 67,385

Tamias minimus, 120 striatus, 271,389

Tamiasciurus hudsonicus, 120,268,303,329,389

Tamiasciurus hudsonicus, in a Secondary Hardwood Forest of Southern Ontario, Cavity Nest Materials of Northern Flying Squirrels, Glaucomys sabrinus, and North American Red Squirrels, 303

Tanacetum parthenium, 44

Taraxacum officinale, 77,182

Taxidea taxus, 150

Taylor, B.R., 76

Tea, Labrador, 81 New Jersey, 203

Teal, Blue-winged, 245

Green-winged, 325

Tear-Thumb, Arrowleaved, 184

Tern, Aleutian, 325

Arctic, 289,433

Black, 50

Caspian, 289

Common, 275,288,433

Forster's, 50

Terrapin, Diamondback, 178

Teucrium canadense ssp. canadense, 185

Texas, Swift Fox, Vulpes velox, Den Use Patterns in Northwestern, 71

Thamnophis, 210 sirtalis, 210 sirtalis pallidulus, 210 sirtalis parietalis, 210 sirtalis sirtalis, 210

Thamnophis sirtalis pallidulus, in Dartmouth, Nova Scotia, Canada, Arboreal Late Summer Courtship Behaviour of Maritime Garter Snake, 210

Thiel, R.P., 256

Thimbleweed, Long-head, 203

Thistle, Canada, 184,302

Thlaspi arvense, 78,185

Thrasher, Brown, 243 
Thuja occidentalis, 304,385,431

Thumb, Lady's, 184

Thymelicus lineola, 182

Tiarella trifoliata, 24

Tilia americana, 256

Timothy, 182

Toadflax, Bastard, 203

Tobacco, Indian, 184

Towhee, Spotted, 244

Toxicodendron rydbergii, 204

Toxostoma rufum, 244

Tragopogon dubius, 185 pratensis ssp. pratensis, 185

Tree, Spindle, 85

Trichodectes canis, 431

Trifolium aureum, 185 campestre, 185 hybridum, 185 pratense, 185

Trirhabda, 182 virgata, 182

Tritomaria quinquedentata, 27

Troglodytes aedon, 244

Trout, Bull, 437

Trout, Salvelinus confluentus, and North American Porcupine, Erethizon dorsatum, Interaction in the Mackenzie Mountains, Northwest Territories, Bull, 437

Tsuga canadensis, 304 heterophylla, 24

Turdus caleanops, 216 migratorius, 216,244

Turdus migratorius, and a Five-lined Skink, Eumeces fasciatus, Predator-Prey Interaction Between an American Robin, 216

Turkey, Wild, 245

Turtle, Blanding's, 178

Common Map, 178

Musk, 178

Painted, 178

Snapping, 130,178

Spiny Softshell, 178

Spotted, 178

Wood, 178

Turtle, Chelydra serpentina, Hatchlings in New York and New Hampshire, Post-Emergence Movements and Overwintering of Snapping, 178

Tympanuchus phasianellus, 245

Typha angustifolia, 53 latifolia, 53,166 $\times$ glauca, 53

Tyrannus tyrannus, 244 verticalis, 244

Ulmus americana, 385

Ultsch, G.R., M. Draud, and B. Wicklow. Post-Emergence Movements and Overwintering of Snapping Turtle, Chelydra serpentina, Hatchlings in New York and New Hampshire, 178

Umbellularia californica, 305

United States and Canada, Human-assisted Movements of Raccoons, Procyon lotor, and Opossums, Didelphis virginiana, between the, 212

Uria aalge, 275,289

Ursus americanus, 120,156,266,330,362,387,430 arctos, 120,156 maritimus, 120

Usnea sp., 18

Vahlodea atropurpurea, 24

Vallisneria, 164 americana, 93

Van Deelen, T.R., 333

Vanderhoff, E.N. Predator-Prey Interaction Between an American Robin, Turdus migratorius, and a Fivelined Skink, Eumeces fasciatus, 216

Van Dyke, F. Colonization of Non-Traditional Range in Dispersing Elk, Cervus elaphus nelsoni, Populations, 133

Veratrum eschscholtzii, 24 viride, 421

Verbascum thapsus, 185

Verbena hastata, 185

Vermont, A High Elevation Record of the Star-nosed Mole (Condylura cristata) in Northeastern, 206

Vermont Reptile and Amphibian Atlas Project Publishes the First Vermont Amphibian Posters, The, 453

Veronica persica, 79 serpyllifolia $\mathrm{ssp}$. serpyllifolia, 185

Vervain, Blue, 185

Vetch, 185

Common, 185

Cow, 185

Slender, 185

Viburnum spp., 359

Vicia cracca, 185 sativa $\mathrm{ssp}$. nigra, 185 tetrasperma, 185

Viola sp., 185 adunca var. adunca, 204 praemorsa, 41 sagittata var. ovata, 204

Violet, 185 tricolor, 79

Arrow-leaf, 204

Hook-spur, 204

Vitis riparia, 204

Vole, 385

Eastern Heather, 120

Gray-tailed, 416

Long-tailed, 120

Meadow, 120,390,416

Northern Red-backed, 120

Red-backed, 271

Root, 120

Singing, 120

Southern Red-backed, 120,206,390

Taiga, 120

Yellow-nosed, 206

Vulpes lagopus, 120

macrotis, 71

velox, 71

vulpes, 73,120,150,271,322,386

Vulpes velox, Den Use Patterns in Northwestern Texas, Swift Fox, 71

Wahoo, Eastern, 85

Western, 85

Wallaby, Red-necked, 268

Walrus, 385

Atlantic, 389 
Wang, G.G., 191

Warbler, Yellow, 244

Waters, N., 155

Waterweed, Canada, 164

Waxwing, Cedar, 244

Way, J.G. Social and Play Behavior in a Wild Eastern Coyote, Canis latrans, Pack, 397

Way, J.G. Movements of Transient Coyotes, Canis latrans, in Urbanized Eastern Massachusetts, 364

Weasel, 385

Least, 120

Short-tailed, 388

Weed, Joe Pye, 184

Wheat, American Cow, 203

Whiskeychan, S., 67

Wicklow, B., 178

Wigeon, 376 American, 245,325

Wilkinson, S.F., P.T. Gregory, C. Engelstoft, and K.J. Nelson. The Rumsfeld Paradigm: Knowns and Unknowns in Characterizing Habitats Used by the Endangered Sharp-tailed Snake, Contia tenuis, in Southwestern British Columbia, 142

Willow, 81,359

Narrow-leaved, 166

Prairie, 204

Winn, L.S., 430

Wintercress, 184

Wisconsin, A Late Born White-tailed Deer, Odocoileus virginianus, Fawn in Southcentral, 333

Wolf, 120,156,256,360,364,385,397,437

Gray [Grey], 386,430

Red, 430

Wolf, Canis lupus, Behavior in Areas of Frequent Human Activity, 256

\section{Index to Book Reviews}

\section{Botany}

Fuliang, C. Ginkgo in China, 445

Hinds, J.W. and P.L. Hinds. The Macrolichens of New England, 345

Kuo, M. MushroomExpert.Com website, 446

Nadkarni, N.M. Between Earth and Sky: Our Intimate Connections to Trees, 447

Shaw, R.B. Grasses of Colorado, 344

Walker, M. Wild Plants of Eastern Canada, 346

Weiming, Z. and X. Zhengchun et al. Exploitation and Utilization of Chinese Spice Plant Resources, 225

Zhongzhen, Z., X. Peigen. Comprehensive Monograph of Contemporary Medicinal Plants (Volumes I-IV), 445

\section{Environment}

De Roy, T. and M. Jones. New Zealand - A Natural History, 109

Honglie, S. China Ecosystems, 108

\section{Miscellaneous}

Adler, K. Contributions to the History of Herpetology Volume 2, 448

Barr, W. Arctic Hell Ship, 226

Beagle Ristaino, J. Pioneering Women in Plant Pathology, 230
Wolf, Canis lupus, Pups into Another Wolf Pack, Attempt to Cross-Foster Gray, 430

Wolverine, $120,156,437$

Woodchuck, 120

Woodley, E., Review by, 113

Woodrat, Bushy-tailed, 120

Worm, Meningeal, 63

Wormwood, Pacific, 203

Wren, House, 244

Marsh, 244

Sedge, 244

Wydeven, A.P., 430

Xanthocephalus xanthocephalus, 245

Xanthoria fallax, 18

hasseana, 18

polycarpa, 21

Yarrow, 184

Yellowthroat, Common, 244

Yukon, Discovery of a Possibly Relict Outbreeding Morphotype of Sparrow's-egg Lady's-slipper Orchid, Cypripedium passerinum, in Southwestern, 295

Yukon, Northern Range Extension of the Pygmy Shrew, Sorex hoyi, in the, 94

Yukon Territory: A Review, Diversity and Distribution of the Terrestrial Mammals of the, 119

Zannichellia palustris, 166

Zapus hudsonius, 120,271,390 princeps, 120

Zenaida macroura, 245,436

Zenaida macroura, Fish-line Entanglement of Nesting Mourning Dove, 436

Zostera marina, 164

Bleakney, J.S. Sods, Soil, and Spades: The Acadians at Grand Pre and their Dykeland Legacy, 347

Calhoun, A.J.K. and P.G. deMaynadier. Science and Conservation of Vernal Pools in Northeastern North America, 448

Erritzoe, J., K. Kampp, K. Winker and C.B. Frith. The Ornithologist's Dictionary, 229

Houston, S., T. Ball and M. Houston. Eighteenth-century Naturalists of Hudson Bay, 227

Jenkins, P. Beneath My Feet: The Memoirs of George Mercer Dawson, 110

Rong, J. The Wolf Totem, 111

Rosenthal, E.J. Birdwatcher: The Life of Roger Tory Peterson, 230

Sandlos, J. Hunters at the Margin: Native People and Wildlife Conservation in the Northwest Territories, 228

\section{Zoology}

Backhouse, F. Owls of North America, 341

Bartlett, R.C. and P.P. Bartlett. Guides and Reference to the (1) Snakes, (2) Crocodilians, Turtles, and Lizards (3) Amphibians, of Eastern and Central North America (North of Mexico), 444

Bergerud, A.T., S.N. Luttich, and L. Camps. The Return of Caribou to Ungava, 220 
Bildstein, K.L., J.P. Smith, E.R. Inzunza, and R.R. Veit. State of North America's Birds of Prey, 339

Bronaugh, W. Wildlife of North America: A Naturalist's Lifelist, 343

Cadman, M.D., D.A. Sutherland, G.G. Beck, D. Lepage, A.R. Couturier. Atlas of Breeding Birds of Ontario 2001-2005, 219

Chester, S. A Wildlife Guide to Chile, 337

Coad, B. Brian Coad's Ichthyology Site, 224

Couzens, D. Extreme Birds, 440

Crump, M. Headless Males Make Great Lovers and Other Unusual Natural Histories, 339

Donnelly, M.A., B.I. Crother, C. Guyer, M.H. Wake, and M.E. White. Ecology \& Evolution in the Tropics: A Herpetological Perspective, 338

Duncan, J. Owls of the World. 103

Fisher, C., A. Joynt, and R.J. Brooks. Reptiles and Amphibians of Canada, 443

Folkens, P.A. Marine Mammals of the Pacific Northwest: A Concise and Comprehensive Waterproof Guide, 442

Fontaine, P.-H. Whales and Seals Biology and Ecology, 107

Franklin, C.J. Turtles: An Extraordinary Natural History 245 Million Years in the Making, 106

Gibbs, J.P., A.R. Breisch, P.K. Ducey, G. Johnson, J.L. Behler, and R.C. Bothner. The Amphibians and Reptiles of New York State: Identification, Natural History, and Conservation, 439

Hughes, J.M. Cranes: A Natural History of a Bird in Crisis, 441

Lecointre, G. and H. Le Guyader. The Tree of Life: A Phylogenetic Classification, 342
Leighton, A.L., J. Hay, C.S. Houston, J.F. Roy, and S. Shadick. Birds of the Saskatoon Area, 223

Lynch, W. Owls of the United States and Canada: A Complete Guide to Their Biology and Behavior, 340

Matsuda, B.M., D.M. Green, and P.T. Gregory. Amphibians and Reptiles of British Columbia, 100

McPhail, J.D. The Freshwater Fishes of British Columbia, 102

Merrick, J.R., M. Archer, G.M. Hickey, and M.S.Y. Lee. Evolution and Biogeography of Australasian Vertebrates, 336

Nudds, J.R. and P.A. Selden. Fossil Ecosystems of North America: A Guide to the Sites and Their Extraordinary Biotas, 338

Schulenberg, T.S., D.F. Stotz, D.F. Lane, J.P. Ołrquote Neill, and T.A. Parker III. Birds of Peru, 101

Seburn, C.N.L. and C.A. Bishop. Ecology, Conservation, and Status of Reptiles in Canada. 104

Smith, A. and Y. Xie. A Guide to the Mammals of China, 222

Steyermark, A.C., M.S. Finkler, and R.J. Brooks. Biology of the Snapping Turtle (Chelydra serpentina), 343

Titlow, B. Seashells: Jewels from the Ocean, 105

Wells, K.D. The Ecology \& Behavior of Amphibians, 439

Wolff, J.O. and P.W. Sherman. Rodent Societies: An Ecological \& Evolutionary Perspective, 105

Zwickel, F.C. and J.F. Bendell. Blue Grouse: Their Biology and Natural History, 101

\section{Young Naturalists}

Meyrick, J. Gracie. The Public Gardens Duck. 113

O'Donnell, L. Amazing Animal Journeys, 449

Whitelaw, I. Snow Dogs! Racers of the North, 449 